\title{
A practical philosophy
}

\author{
The expanding biotechnology landscape offers new intersections with traditional strengths in \\ chemical biology.
}

Chemical biology relies on a dynamic interplay between basic science and 'applied' chemical biology, in which the development and improvement of techniques and tools with practical applications in biology in turn advance and inform basic research. For example, high-throughput and high-content screening coupled with careful biochemical and cellular analyses have generated useful chemical probes of many biological systems. Advanced imaging reagents have enabled investigations into cellular processes with outstanding spatial and temporal precision. New strategies to synthesize or modify biomolecules have been used to define specific molecular behaviors and functions. This relationship between fundamentals and practice is shared with industrial biotechnology in its aim and its growing capacity to achieve real-world objectives and to deliver a 'bio-based society'.

Scientists working in the fields of metabolic engineering, synthetic biology and biocatalysis-topics squarely at the interface of chemistry and biology_have already been pivotal in developing new strategies and ideas that are driving white biotechnology forward. Indeed, for many chemical biologists the word 'biotechnology' calls to mind reports such as the engineered microbial production of artemisinic acid (Ro, D.-K. et al. Nature 440, 940-943, 2006) as a cost-effective strategy to treat malaria, or the increased titers of isoprene that are moving Genencor into a unique partnership with tire manufacturers (Whited, G.M. et al. Ind. Biotechnol. 6, 152$163,2010)$. These stories, which have relied on intimate chemical biology knowledge of biosynthesis and metabolism coupled with the engineering concepts and methodologies of industrial biotechnology, illustrate the great potential synergies between these two scientific areas.

To optimize these interactions, chemical biologists must become aware of the scientific challenges facing the biotechnology industry and determine how they can best contribute. Those whose strengths lie in unraveling and understanding biological systems at the molecular level will advance the field best by adding to the fundamental knowledge that underpins biotechnological applications. For example, plant biologists' investigations into vacuolar storage of vitamins will inform advances in creating nutritionally superior foods. Carbohydrate chemists' growing understanding of the function of glycosyltransferases in constructing cell walls can be co-opted to speed biomass breakdown. Biochemists and biophysicists will provide important calibrations of metabolic models with their in vivo analyses of protein folding and enzyme kinetics. These basic mechanistic studies thus supply critical information that will serve as intellectual feedstock for practical industrial biotechnological applications.

Other chemical biologists are driven by a desire to create enabling tools and methods and have made available a wealth of functional switches, quantitative readouts and bioorthogonal chemistries. Scientists with this goal should seek out information and advice about specific needs in the field and aim for simple, general and robust methods that address open technical challenges. For example, though metabolomics has already provided exciting new insights into cellular function, the field is lacking a reliable and general technique to 'quench' metabolism so that the readout matches the cellular environment the experiment was intended to probe. Conversations with colleagues can help to identify these kinds of technical gaps. Information from relevant organizations, including the recent report on biorefineriesone-stop factories converting biomass into target products-issued by the World Economic Forum (http://bit.ly/cxQ54Z), can also help to define current technological capabilities and open scientific questions. Finally, even those chemical biologists working on basic studies should be mindful of the potential applications of their work; in many cases, even slight refocusing or development of a fundamental research project could yield important practical outcomes.

Scientists who want to more directly apply chemical biology knowledge to advance industrial biotechnology goals should first educate themselves about how their work can achieve broader impact. Numerous resources can help identify established priorities in the field, such as the 'top' chemicals obtainable from biomass conversion, listed in a 2004 report by the United States Department of Energy (http://bit.ly/bcPGMC). News sources and forums for original research, such as our sister journal Nature Biotechnology, can also lead the way to new opportunities. Once acclimated to the biotechnology terrain, chemical biologists may find that their abilities to navigate between scientific disciplines mean that their greatest potential contribution is in identifying creative new strategies. For example, research from the lab of James Liao-this year's recipient of the Presidential Green Chemistry Challenge Award (http://bit.ly/c58fD0) - provides an elegant example of using $\mathrm{CO}_{2}$ as an alternative carbon source for biofuel production.

Chemical biologists wishing to expand their interests in white biotechnology should also educate themselves in the complex political, ethical and regulatory considerations facing the field. In particular, although the commercial opportunities and societal concerns driving industrial biotechnology are clear, the rules that will govern this burgeoning field lag behind. Companies, nations and individual researchers are stakeholders in the science, but although scientists increasingly collaborate across national borders, individual countries will have different priorities and capabilities in developing laws and regulations and may have less impetus to come to agreement. Businesses in this sector tout their interest in cooperative efforts and open innovation, but there is little precedent for what will happen when partnerships collide with profit. With this in mind, the recent meetings of the first World Council on Industrial Biotechnology (http://www.whitebt.org/) and the Global Advanced Biofuels Scale Up Summit 2010 (http://www.advanced-biofuels-scale-up. $\mathrm{com} /$ ) provided an important step towards defining clear expectations and speeding translation from test tube to production plant.

Whether engaged in these practical ventures as scientists, CEOs or citizens, all parties must participate in the conversation about biotechnology, for it is clear that the bio-based society is quickly coming of age. Bioethanol and other biofuels are changing the way land is used and engines are designed. Engineered bacteria are increasing production of useful commodity chemicals, ranging from amino acids to polymer precursors. A growing number of production-scale biorefineries are under construction and in operation. As chemical biologists step forward to contribute, we look forward to exciting new fundamental discoveries and applications capable of converting bioproducts from fiction into fact. 